\title{
A METHOD FOR GENERATING FOREST/NON-FOREST MAPS FROM TANDEM-X INTERFEROMETRIC DATA
}

\author{
Michele Martone, Paola Rizzoli, Benjamin Bräutigam, and Gerhard Krieger
}

German Aerospace Center (DLR), Microwaves and Radar Institute, Oberpfaffenhofen, Germany

\begin{abstract}
In this paper a method for the generation of a global forest/nonforest map from TanDEM-X interferometric data is presented. The quality of interferometric products is strongly influenced by the specific characteristics of the illuminated land cover type. Over forested areas the presence of multiple scatterers at different heights and within a single resolution cell results in an increase of the interferometric phase uncertainty (the so-called volume decorrelation), whose intensity depends on several factors, such as the acquisition geometry, the sensor parameters, and the canopy density. From each TanDEM-X coherence map the volume decorrelation contribution can be estimated, leading to the derivation of a forest/non-forest map. This paper shows the developed approach for discriminating between forested and non-forested areas from TanDEM-X interferometric data, and presents some examples aimed at verifying the validity of the proposed method. From this, mosaics can be generated from the TanDEM-X quicklook data set at a final resolution up to $25 \mathrm{~m} \times 25 \mathrm{~m}$.
\end{abstract}

Index Terms - TanDEM-X, SAR interferometry (InSAR), classification, forest map.

\section{INTRODUCTION}

Land cover classification by means of remote sensing data is of fundamental importance for a broad range of commercial and scientific uses. In particular, the detection of vegetated areas is of great interest for global change research and for applications in agriculture, cartography, geology, forestry, as well as regional planning. At a global level, only in the very last years high-resolution forest classification maps have been produced using global mosaics of multi-spectral Landsat sensor data, together with forest cover change maps from 2000 to 2012 at $30 \mathrm{~m}$ resolution [1]. Recently, the Advanced Land Observing Satellite (ALOS) L-band synthetic aperture radar (SAR) has produced forest maps with $25 \mathrm{~m}$ spacing from global SAR amplitude mosaics collected from 2007 to 2010 [2]. This paper presents an approach to generate a global forest/non-forest map from TanDEM-X interferometric SAR

The TanDEM-X project is partly funded by the German Federal Ministry for Economic Affairs and Energy (Förderkennzeichen $50 \mathrm{EE} 1035$ ). data. The TanDEM-X mission comprises the two twin satellites TerraSAR-X (TSX) and TanDEM-X (TDX). Since October 2010 TSX and TDX fly in close orbit formation of a few hundred meters distance and act as a large single-pass SAR interferometer (InSAR), with the main goal of producing a global and consistent Digital Elevation Model (DEM) with an unprecedented accuracy [3].

In the next section some theoretical background is recalled and the approach for the discrimination between forested and non-forested map is developed. Then, some examples over different forest types, aimed at verifying the proposed method, are presented. Further aspects and issues to be taken into account for the generation of a global and consistent forest/non-forest map from TanDEM-X interferometric data are summarized in Section 4.

\section{ESTIMATION APPROACH}

The key quantity used for the assessment of InSAR performance is the correlation coefficient between master and slave acquisitions. Its modulus represents the interferometric coherence $\gamma$, which describes the amount of noise affecting the interferogram. Several error sources contribute to a coherence loss in TanDEM-X interferometric data [4], which, assuming statistical independence, can be factorized as follows:

$$
\gamma=\gamma_{\mathrm{SNR}} \cdot \gamma_{\mathrm{Quant}} \cdot \gamma_{\mathrm{Amb}} \cdot \gamma_{\text {Range }} \cdot \gamma_{\mathrm{Azimuth}} \cdot \gamma_{\mathrm{Temp}} \cdot \gamma_{\mathrm{Vol}} \cdot
$$

The terms on the right-hand side describe decorrelation due to: limited signal-to-noise ratio $\left(\gamma_{\mathrm{SNR}}\right)$, quantization errors $\left(\gamma_{\text {Quant }}\right)$, ambiguities $\left(\gamma_{\mathrm{Amb}}\right)$, baseline decorrelation $\left(\gamma_{\text {Range }}\right)$, errors due to relative shift of Doppler spectra $\left(\gamma_{\text {Azimuth }}\right)$, and temporal decorrelation $\left(\gamma_{\text {Temp }}\right)$. The last term $\left(\gamma_{\text {Vol }}\right)$ describes the coherence loss caused by volume scattering, and represents the contribution which is predominantly affected by the presence of vegetation. Given a coherence estimate $\gamma$, it is straightforward to quantify the volume decorrelation contribution as

$$
\gamma_{\text {Vol }}=\frac{\gamma}{\gamma_{\text {SNR }} \cdot \gamma_{\text {Quant }} \cdot \gamma_{\text {Amb }} \cdot \gamma_{\text {Range }} \cdot \gamma_{\text {Azimuth }} \cdot \gamma_{\text {Temp }}}
$$

The impact of all decorrelation sources on TanDEM-X data is shortly discussed in the following: 
- $\gamma_{\mathrm{SNR}}$ represents the coherence loss due to the finite sensitivity of the receiving antenna. For TanDEM-X, an estimation of the signal-to-noise ratio (SNR) is obtained, for each pixel, from the measured backscatter coefficient and the noise equivalent sigma zero (NESZ), which describes the system noise floor and depends on the antenna pattern, the instrument thermal noise, and the processing filters. Given an SNR value and assuming same performance for both master and slave channels, one obtains

$$
\gamma_{\mathrm{SNR}} \cong \frac{1}{1+\mathrm{SNR}^{-1}}
$$

For TanDEM-X nominal acquisitions, $\gamma_{\mathrm{SNR}}>0.8$ can be expected for most types of land coverage [4].

- $\gamma_{\text {Quant }}$ describes the errors due to the quantization of the recorded raw data signals. On TSX and TDX, block adaptive quantization (BAQ) is employed for raw data compression. In Fig. 1 the mean coherence loss for different quantization rates is expressed as a function of the total coherence. This look-up-table has been derived from real TanDEM-X data commanded with BAQ bypass and recompressed to the available quantization rates [5].

- TanDEM-X operates in single-pass bistatic mode, which means that either TSX or TDX is used for transmission and both satellites record simultaneously the backscattered signal from the Earth's surface. In this scenario, the coherence loss caused by a temporal change in the scatterer structure can be reasonably neglected (i.e. $\gamma_{\text {Temp }}=1$ ).

- Regarding the remaining error contributions $\gamma_{\mathrm{Amb}}$, $\gamma_{\text {Range }}$, and $\gamma_{\text {Azimuth }}$, adaptive selection of the azimuth processing bandwidth as well as total independent zero Doppler steering are employed in TanDEM-X, and an additional coherence loss in the order of about $2 \%$ can be reasonably assumed [3], [4].

Once the volume decorrelation contribution has been estimated from (2), it has to be properly associated to a forest/non-forest classification. To do this, the theoretical coherence can be derived from the ensemble average over all the scatterers constituting the volume [3]

$$
\gamma_{\text {Vol,Theor }}=\frac{\int_{0}^{h_{v}} \sigma^{0}(z) \cdot \exp \left(j 2 \pi \frac{z}{\mathrm{HoA}}\right) \mathrm{d} z}{\int_{0}^{h_{v}} \sigma^{0}(z) \cdot \mathrm{d} z},
$$

where $h_{v}$ indicates the volume height, HoA is the height of ambiguity (i.e. the height difference corresponding to a complete $2 \pi$ cycle of the interferometric phase), and $\sigma^{0}(z)$ represents the vertical scattering profile, which, by assuming the extinction in a homogeneous medium, is modeled as

$$
\sigma^{0}(z)=\exp \left[-2 \cdot \beta \cdot \frac{h_{v}-z}{\cos \left(\theta_{\mathrm{i}}\right)}\right],
$$

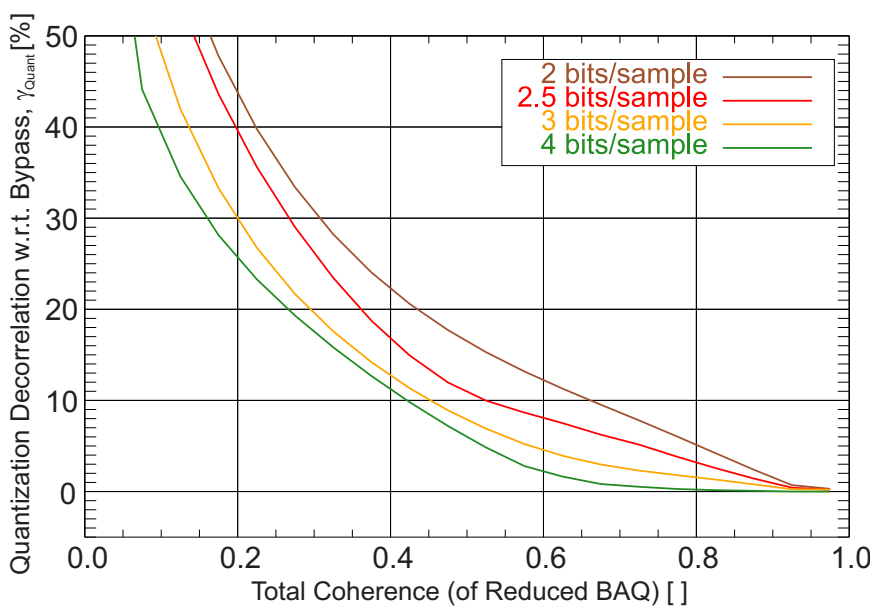

Fig. 1. Mean coherence loss for different quantization rates expressed as a function of the total coherence. This lookup-table has been derived from real TanDEM-X data commanded with BAQ bypass and recompressed using different $\mathrm{BAQ}$ rates [5].

being $\theta_{\mathrm{i}}$ the local incidence angle and $\beta$ the one-way amplitude extinction coefficient. Fig. 2 shows the volume decorrelation $\gamma_{\text {Vol }}$ over the height of ambiguity estimated from repeated bistatic acquisitions over a test area in the Amazon rainforest, with an incidence angle of $30^{\circ}$ (blue circles) and $48^{\circ}$ (in red). The theoretical volume decorrelation $\gamma_{\text {Vol,Theor }}$ is depicted for the $30^{\circ}$ (turquoise line), and for the $48^{\circ}$ case (in brown) as well. The height of the trees has been derived from the corresponding DEMs as the height difference between vegetated areas and clear-cuts, and is of about $35 \mathrm{~m}$. A minimum mean square error estimation has been performed to derive the extinction coefficient, and a $\beta$ of around $0.4 \mathrm{~dB} / \mathrm{m}$ has been found for both time series, which is in good agreement with previous publications [6]. In both cases, we obtained a correlation $r^{2}$ larger than 0.95. From Fig. 2 it can be clearly seen that the specific acquisition geometry plays a key-role in determining the influence of volume decorrelation phenomena on interferometric SAR data, for which sufficiently large HoAs (i.e. smaller baselines) are preferred. For TanDEM-X, the heights of ambiguity used for the generation of the global DEM are in the range between 30 and $100 \mathrm{~m}$.

Thus, depending on the specific height of ambiguity and incidence angle, we may define volume decorrelation regions which identify forested or non-forested areas, as shown in Fig. 3. In the present approach, volumes with a height between 10 (orange line) and 100 (red line) meters are classified as "forest", according to (4). For the two curves an extinction of $0.5 \mathrm{~dB} / \mathrm{m}$ and $0.2 \mathrm{~dB} / \mathrm{m}$ have been considered, respectively, in order take into account the heterogeneity of the forest scattering volume [6], and to include a wider spectrum of forest types. If on the one hand volume decorrelation strongly de- 


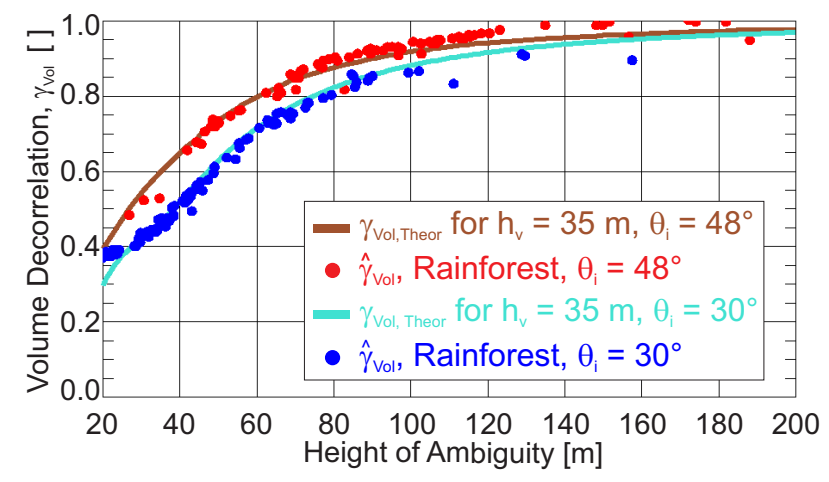

Fig. 2. Volume decorrelation $\gamma_{\mathrm{Vol}}$ over height of ambiguity estimated from repeated TanDEM-X acquisitions over a test area in the Amazon rainforest, with incidence angles of $30^{\circ}$ (blue circles) and $48^{\circ}$ (in red). The expected $\gamma_{\text {Vol,Theor }}$ is depicted for the steeper incidence angle (turquoise line), and for the shallower one (in brown) as well. In both cases $\beta$ of about $0.4 \mathrm{~dB} / \mathrm{m}$ has been estimated, and a correlation $r^{2}$ larger than 0.95 has been verified.

pends on the canopy density and height, high-frequency microwaves are on the other hand very sensitive to the presence of vegetation, due to the limited penetration capability of Xband waves. Therefore, their ability in distinguishing woody and herbaceous vegetation may be more limited.

\section{FOREST CLASSIFICATION FROM SINGLE TANDEM-X ACQUISITIONS: SOME EXAMPLES}

On the left-hand side of Fig. 4 forest (represented in green) and non-forest (in brown) areas classified according to the present method are depicted for a bistatic TanDEM-X acquisition over a forested region in Bavaria, Germany, overlaid on a GoogleEarth optical image. On the right-hand side of the figure the optical image alone is given for comparison (the area of interest is delimited by the red rectangle). In Fig. 5 a forest/non-forest map (and the corresponding optical image) is depicted for an area over Amazon rainforest. The forest classification for the region indicated in the blue rectangle is shown in Fig. 6. In general, the different land cover types are correctly discriminated, even for the case of narrow forested (bottom-left of Fig. 6) and non-forested (mid-upper part of the figure) passages of few tens of meters width, which verifies the potentials of the proposed approach. On the very top of Fig. 6 there are small patches that still look vegetated but are classified as "non-forest". However, if looking closer at the optical image, one can notice that they correspond effectively to a different vegetation type. It is clear that a consistent validation by means of land cover classification is indispensable for a quantitative evaluation of the obtained results, and which will be object of future studies.

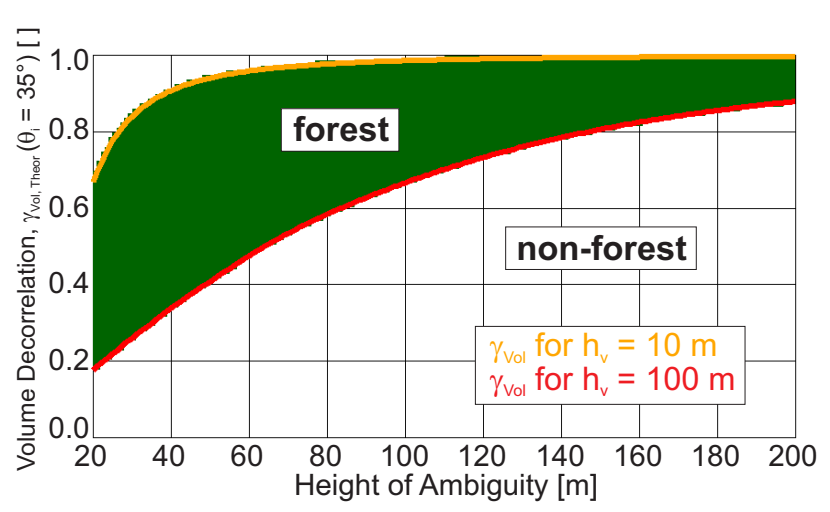

Fig. 3. Volume decorrelation ranges associated to forest (green region) and non-forest, as a function of the height of ambiguity, for an incidence angle $\theta_{\mathrm{i}}=35^{\circ}$. For the present classification, forest are supposed to be characterized by trees with a height between 10 (orange line, $\beta=0.5 \mathrm{~dB} / \mathrm{m}$ ) and 100 (red line, $\beta=0.2 \mathrm{~dB} / \mathrm{m}$ ) meters.

\section{OUTLOOK ON A GLOBAL FOREST/NON-FOREST MAP: ISSUES AND POTENTIALS}

Since the beginning of the TanDEM-X mission (end of 2010), about half a million high-resolution bistatic InSAR scenes have been acquired covering all the Earth's land masses. A single bistatic scene has typically a ground extension of about $30 \mathrm{~km}$ in range by $50 \mathrm{~km}$ in azimuth. From each of these, quicklook images for several SAR and InSAR measures are generated as by-products from the operational interferometric processing chain. These quicklooks are obtained by applying a spatial averaging process to the corresponding operational TanDEM-X interferometric data in full resolution, like the coherence matrix or the roughly calibrated DEM. They have a ground pixel spacing of about $25 \mathrm{~m} \times 25 \mathrm{~m}$, and are taken into account as input data base for generating quicklook mosaics. All images were acquired in bistatic stripmap mode and single horizontal polarization, covering the entire range of incidence angles of the nominal TanDEM-X mission (between $29^{\circ}$ and $49^{\circ}$ ) [3]. The global land masses have been acquired at least twice. In order to further improve the performance, many densely forested areas have been covered up to four times in a time frame of several years, which can be exploited for detecting possible changes in the forest cover. Global mosaics from TanDEM-X quicklook data have been generated and exploited for continuous performance monitoring as well as for optimization of the acquisition strategy during the mission [7]. For the generation of a global and consistent forest/non-forest map from TanDEM-X interferometric data, several issues need to be taken into account, such as, for example, the identification of shadow and layover areas in presence of rugged terrain, as well as the recognition of urban areas, to be opportunely filtered out. For these purposes 

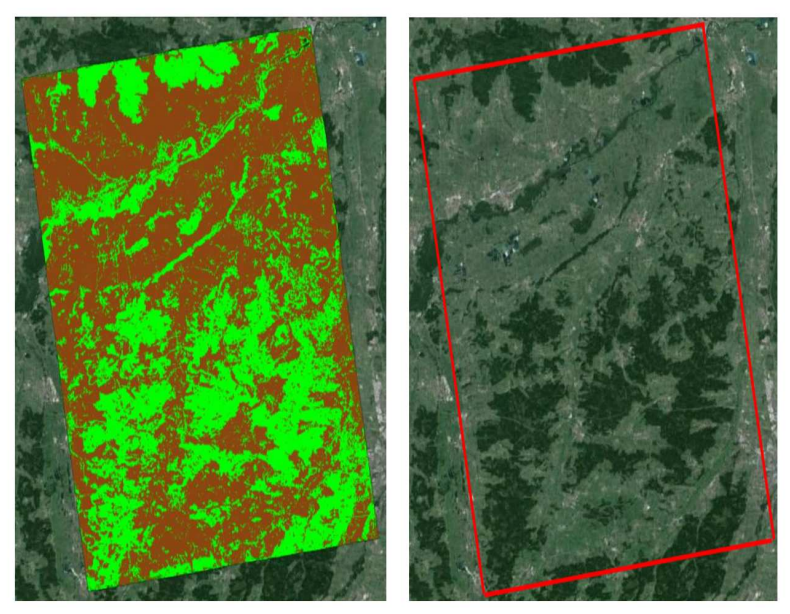

Fig. 4. (Left) Forest/non-forest classification map (depicted in green and brown, respectively) of a forested area located in the Bavaria region, Germany, overlaid on a GoogleEarth optical image, which is given on the right-hand side for comparison. The considered area is delimited by the red rectangle, and extends by about $50 \mathrm{~km} \times 30 \mathrm{~km}$.

additional information will have to be exploited, such as the SAR image amplitude, the DEM height and the local slope, as well as the availability of multiple coverages with different baselines [3], which will allow to further improve the classification capabilities of TanDEM-X interferometric data. In this sense, the analyses in this paper are a first step for the generation of such a kind of products.

\section{REFERENCES}

[1] "Global Forest Change," http:// earthenginepartners.appspot.com/ science-2013-global-forest, [Online; accessed 2013].

[2] M. Shimada, T. Itoh, T. Motooka, M. Watanabe, T. Shiraishi, R. Thapa, and R. Lucas, "Newglobal forest/nonforest maps from ALOS PALSAR data (20072010)," Remote Sens. of Env., vol. 155, pp. 13-31, May 2014.

[3] G. Krieger, A. Moreira, H. Fiedler, I. Hajnsek, M. Werner, M. Younis, and M. Zink, "TanDEM-X: A satellite formation for high-resolution SAR interferometry," IEEE Trans. Geosci. Remote Sens., vol. 45, no. 11, pp. 3317-3341, November 2007.

[4] M. Martone, B. Bräutigam, P. Rizzoli, C. Gonzalez, M. Bachmann, and G. Krieger, "Coherence evaluation of TanDEM-X interferometric data," ISPRS J. of Photogr. Remote Sens., vol. 73, pp. 21-29, September 2012.
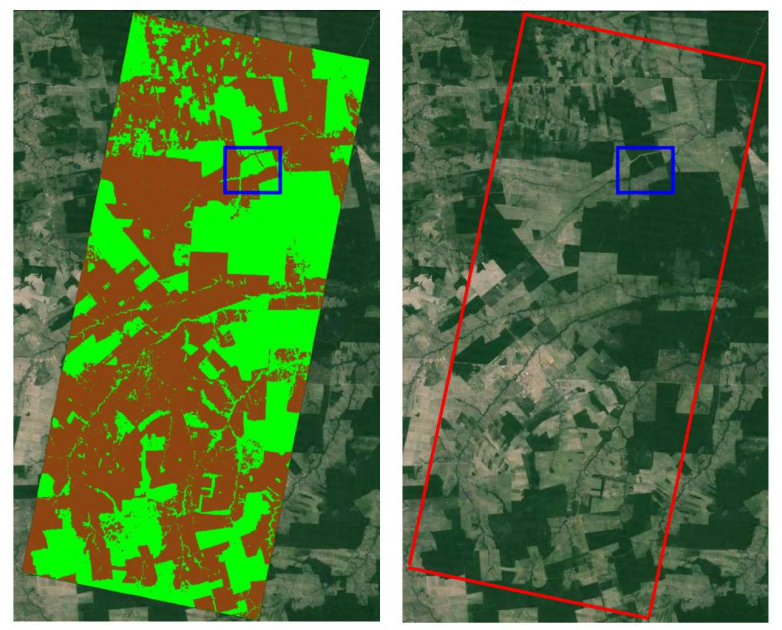

Fig. 5. Forest/non-forest classification map (depicted in green and brown, respectively) of an area located in the Amazon rainforest, Brazil, overlaid on a GoogleEarth optical image, which is given on the right-hand side for comparison. The considered area is delimited by the red rectangle and extends by about $55 \mathrm{~km} \times 30 \mathrm{~km}$.
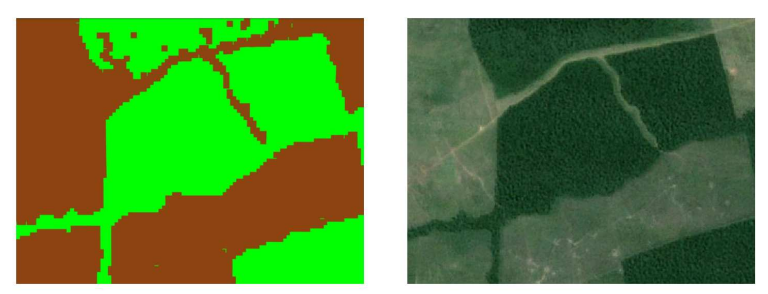

Fig. 6. (Left) Classification map and (right) corresponding GoogleEarth optical image for the region indicated in the blue rectangle of Fig. 5 . The area extends by about $5 \mathrm{~km} \times 6 \mathrm{~km}$, and the resolution is of $25 \mathrm{~m} \times 25 \mathrm{~m}$.

[5] M. Martone, B. Bräutigam, and G. Krieger, "Quantization effects in tanDEM-X data," IEEE Trans. Geosci. Remote Sens., vol. 53, no. 2, pp. 583-597, February 2015.

[6] J. Praks, O. Antropov, and M. T. Hallikainen, "LIDARaided SAR interferometry studies in boreal forest: scattering phase center and extinction coefficient at X- and L-band," IEEE Trans. Geosci. Remote Sens., vol. 50, no. 10, pp. 3831-3843, October 2012.

[7] P. Rizzoli, M. Martone, and B. Bräutigam, "Global interferometric coherence maps from TanDEM-X quicklook data," vol. 11, no. 11, pp. 1861-1865, November 2014. 